\title{
Potencial de espécies de Bambu como fonte energética
}

\author{
Bamboo species potential as energy source
}

\author{
Deborah Rodrigues de Souza Santos ${ }^{1}$, Carlos Roberto Sette Junior ${ }^{2}$, \\ Macksuel Fernandes da Silva ${ }^{3}$, Fabio Minoru Yamaji ${ }^{4}$ e Rogério de Araújo Almeida ${ }^{2}$
}

\begin{abstract}
Resumo
O objetivo do presente trabalho foi o de avaliar as características da biomassa e do carvão vegetal das espécies Bambusa tuldoides, Bambusa vulgaris var. vittata e Dendrocalamus asper visando determinar o seu potencial para o uso energético. Para fins comparativos, foram utilizadas árvores de híbridos de Eucalyptus grandis $x$ Eucalyptus urophylla com 6 anos. A biomassa das espécies foi caracterizada (densidade básica, análise imediata, poder calorífico superior e densidade energética) e foi produzido carvão vegetal com taxa de aquecimento de $1,67^{\circ} \mathrm{C} \cdot \mathrm{min}^{-1} \mathrm{e}$ temperatura final de $450^{\circ} \mathrm{C}$ para posterior caracterização (densidade básica, análise imediata, poder calorífico superior, densidade energética e rendimento gravimétrico). As espécies de bambu apresentaram características energéticas similares ou superiores em relação ao híbrido de eucalipto, exceto para os teores de materiais voláteis e de cinzas, indicando o seu potencial para o uso como fonte de energia.
\end{abstract}

Palavras-chave: Bioenergia; Carbonização; Eucalipto

\begin{abstract}
The objective of this research work was to evaluate the characteristics of biomass and charcoal of Bambusa tuldoides, Bambusa vulgaris var. vittata and Dendrocalamus asper in order to investigate its potential for energy use. For comparative purposes, we used trees of Eucalyptus grandis x Eucalyptus urophylla hybrids trees with 6 years were employed. The biomass was characterized (specific gravity, proximate analysis, high heating value and energy density). The charcoal was produced with a heating rate of $1,67^{\circ} \mathrm{C} \cdot \mathrm{min}^{-1}$ and final temperature of $450^{\circ} \mathrm{C}$ for later characterization (specific gravity, proximate analysis, high heating value, energy density and gravimetric yield). The bamboo species showed similar or superior energy characteristics in relation to the eucalyptus hybrid, except for the volatiles and ash content. The bamboo species has energy properties that indicate their potential for use as energy source.
\end{abstract}

Keywords: Bioenergy; Carbonization; Eucalyptus

\section{INTRODUÇÃO}

O setor energético brasileiro é fundamentado no uso de fontes renováveis, sendo a hidráulica a principal fonte de geração de energia elétrica, embora tenha uma redução de 4,5\% de 2013 para 2014. (EPE, 2015). Apesar da característica renovável, a geração de energia a partir dos recursos hídricos é vulnerável, especialmente em períodos prolongados de estiagem, podendo provocar apagões e forçar medidas públicas de economia de energia.

Os cenários atuais e futuros dos efeitos das mudanças climáticas globais preveem alterações nos regimes hídricos em várias regiões do Brasil (NOBRE et al., 2007; IPCC, 2012), podendo provocar impactos na geração de energia hidroelétrica. Neste cenário, a busca por fontes de geração de energia renovável alternativas à geração hidroelétrica torna-se fundamental e a bioenergia está se tornando cada vez mais importante, tanto para a geração de eletricidade como para a produção de biocombustíveis a partir da biomassa (SCHAEFFER et al., 2008).

\footnotetext{
${ }^{1}$ Mestranda em Recursos Florestais. USP - Universidade de São Paulo / ESALQ - Escola Superior de Agricultura "Luiz de Queiroz". Av. Pádua Dias, 11 - Cx. Postal 9 - 13418-900 - Piracicaba - SP. E-mail: deborahr2s.sa@gmail.com.

2Professor Doutor do Departamento de Engenharia Florestal. UFG - Universidade Federal de Goiás. Rodovia Goiânia - Nova Veneza, km 0 - Campus Samambaia - Caixa Postal 131 - 74690-900 - Goiânia, GO, Brasil. E-mail: crsettejr@hotmail.com; rogerioufg@gmail.com.

${ }^{3}$ Mestrado em Agronomia. UFGO - Universidade Federal de Goiás. Rodovia Goiânia - Nova Veneza, km 0 - Campus Samambaia - Caixa Postal 131 - 74690-900 - Goiânia, GO, Brasil. E-mail: macksuelfernandes@florestal.eng.br.

${ }^{4}$ Professor Doutor do Departamento de Engenharia Florestal. UFSCar - Universidade Federal de São Carlos. Rodovia João Leme dos Santos km110 - Itinga- 18052780 - Sorocaba, SP, Brasil. E-mail: fmyamaji@ufscar.br.
}

Sci. For., Piracicaba, v. 44, n. 111, p. 751-758, set. 2016 DOI: dx.doi.org/10.18671/scifor.v44n111.21 
O Brasil possui excelentes condições edafoclimáticas e territoriais para a ampliação da participação da biomassa agrícola e florestal nas suas matrizes energéticas, sendo a madeira e as culturas agrícolas, como a cana de açúcar, as principais fontes energéticas de biomassa utilizadas atualmente no Brasil (SOARES et al., 2015). Contudo, outras fontes de biomassa alternativas com potencial para geração de energia, tais como os resíduos agroflorestais e as gramíneas, têm sido utilizadas e se tornaram foco de estudos na substituição dos combustíveis fósseis e do gás natural.

Dentre estes combustíveis alternativos estão os bambus de espécies lenhosas, cuja elevada taxa de crescimento e produção acelerada de biomassa os colocam em posição de destaque (SCURLOCK et al., 2000).

Nos últimos anos, o aumento do conhecimento e da pesquisa sobre alguns aspectos das espécies de bambu tem tido um impacto econômico significativo, originando novos usos industriais, tais como painéis estruturais e produção de papel e celulose. No entanto, o escasso conhecimento científico sobre as espécies de bambu, especialmente no que se refere à possibilidade de sua utilização como fonte de energia, tem restringindo o pleno desenvolvimento da cultura nesta área. A energia da biomassa de bambu tem grande potencial, uma vez que pode ser processada de várias maneiras (conversão térmica ou bioquímica) para produzir diferentes produtos energéticos (carvão, gás e biocombustíveis), que podem substituir os produtos provenientes dos combustíveis fósseis (TRUONG; LE, 2014).

Neste sentido, ações e projetos de pesquisa que tenham por objetivo avaliar as características energéticas de diferentes espécies de bambu são fundamentais para o desenvolvimento desta importante área. Uma das ações governamentais nesta área foi a instituição da Política Nacional de Incentivo ao Manejo Sustentado e ao Cultivo do Bambu (PNMCB), Lei 12.484 de 2011 (BRASIL, 2011), que tem como objetivo principal o desenvolvimento da cultura do bambu no Brasil, colocando esta cultura como fundamental para o desenvolvimento econômico e social do país. Os trabalhos científicos que avaliaram a produção de carvão vegetal a partir da biomassa de espécies de bambu e suas características energéticas (BRITO et al. 1987; SCURLOCK et al., 2000; COSTA, 2004; VARANDA et al., 2010; MOREIRA, 2012) são escassos, sendo essencial o desenvolvimento de novos projetos de pesquisa face à crescente demanda por energia renovável alternativa e sustentável.

Para a determinação do potencial de espécies de bambu para o uso energético devem ser avaliadas características da biomassa e do carvão vegetal como a química imediata, o poder calorífico, as densidades básica e energética e o rendimento gravimétrico (ARANTES et al., 2013; CARNEIRO et al., 2014; SOARES et al., 2014, 2015) uma vez que estas irão indicar o seu direcionamento para o uso siderúrgico e/ou para a queima direta.

O objetivo do presente trabalho foi o de avaliar as características energéticas da biomassa e do carvão vegetal de espécies de bambu, comparando com um híbrido clonal de eucalipto, visando determinar o seu potencial para o uso energético.

\section{MATERIAL E MÉTODOS}

\section{Seleção, preparo e carbonização das amostras}

Foram selecionados e coletados colmos maduros em área de touceiras experimentais com 5 anos de idade das espécies Bambusa vulgaris Schard. ex J.C. Wendl. var. vittata, Dendrocalamus asper (Schult. \& Schult.) Backer ex. K. Heynek e Bambusa tuldoides Munro, localizadas na região central do Estado de Goiás ( $16^{\circ} 36^{\prime} \mathrm{S}$ e $49^{\circ} 17^{\prime} \mathrm{W}$ ) com altitude de $730 \mathrm{~m}$ e clima do tipo Aw (quente e semi-úmido), segundo classificação de Köppen, com estação seca bem definida nos meses de maio a setembro, precipitação média anual de $1.400 \mathrm{~mm}$ e solo caracterizado como latossolo vermelho escuro.

Para a seleção dos colmos maduros levou-se em consideração os critérios estabelecidos por Hidalgo-Lopez (2003): posicionados no centro da touceira, com elevada dureza e coloração escura. Para cada uma das espécies foram escolhidos e cortados, aleatoriamente, dez colmos maduros em touceiras diferentes e seccionados dois segmentos na altura do DAP $(1,3 \mathrm{~m})$ de cada colmo, na forma de seções circulares com $5 \mathrm{~cm}$ de espessura, totalizando 20 amostras por espécie.

Para fins comparativos, foram selecionadas dez árvores de híbridos de Eucalyptus grandis $x$ Eucalyptus urophylla com 6 anos, de uma plantação florestal experimental (espaçamento 3,0 m x 2,0 $\mathrm{m}$ ) localizada na mesma região das touceiras de bambu e cortados discos de $5 \mathrm{~cm}$ de espessura na 
altura do DAP (1,3 m) para posterior corte de cunhas opostas, conforme sugerido por Carneiro et al. (2014) e Soares et al. (2015), totalizando 20 amostras.

Do total de amostras de cada espécie estudada, (i) dez foram utilizadas para caracterização da biomassa sendo transformadas em serragem utilizando-se um moinho de facas do tipo Willey e submetidas a uma separação mecânica no agitador orbital de peneiras com batidas intermitentes, para a seleção da fração retira na peneira $\mathrm{n}^{\circ} 24$ internacional, com malha de 60 mesh e (ii) dez para a produção e caracterização do carvão vegetal, sendo previamente secas em estufa a $105^{\circ} \mathrm{C}$ e carbonizadas em forno mufla, com dimensões de $60 \mathrm{~cm}$ x $60 \mathrm{~cm}$ x $70 \mathrm{~cm}$ e equipado com um sistema de controle de temperatura e tempo, com taxa de aquecimento de $1,67^{\circ} \mathrm{C} . \mathrm{min}^{-1}$ e temperatura final de $450{ }^{\circ} \mathrm{C}$, permanecendo estabilizado na temperatura final por um período de $30 \mathrm{~min}$ (ASSIS et al., 2012; ARANTES et al., 2013; SOARES et al., 2014, 2015). Após cada carbonização, foi determinado o rendimento gravimétrico em carvão.

\section{Caracterização energética da biomassa e do carvão vegetal}

As análises descritas a seguir foram realizadas tanto na biomassa como no carvão vegetal das espécies estudadas.

Os procedimentos para a análise imediata baseiam-se na norma ABNT NBR 8112 (ABNT, 1983) determinando-se o teor de materiais voláteis, cinzas e carbono fixo.

O poder calorífico superior (PCS) foi determinado por meio de um calorímetro, conforme a Norma ABNT NBR 8633 (ABNT, 1984).

A densidade básica foi determinada de acordo com o método hidrostático, por meio de imersão em água, conforme descrito na norma ABNT NBR 11941 (ABNT, 2003) e a densidade energética foi calculada a partir do produto entre o valor do poder calorífico e a densidade básica.

\section{Análise estatística dos dados}

$\mathrm{Na}$ análise estatística dos resultados foi utilizado o delineamento inteiramente casualizado (DIC) com quatro tratamentos (espécies) e aplicado o programa JMP (SAS INSTITUTE, 1997). Foram aferidos os "outliers" pelo método Box-Plot (TUKEY, 1977), a normalidade de distribuição dos dados pelo método Shapiro-Wilk (SHAPIRO; WILK, 1965) e a heterogeneidade da variância pelos métodos Bartlett (BARTLETT, 1937) e Levene (LEVENE, 1960). Os dados referentes aos resultados das propriedades energéticas da biomassa e do carvão vegetal apresentaram normalidade de distribuição e homogeneidade de variância, sendo então aplicada a análise de variância (ANOVA), verificando o efeito da espécie sobre cada propriedade e aplicado o teste de Tukey, ajustado a 95\% de probabilidade.

\section{RESULTADOS E DISCUSSÃO}

\section{Caracterização energética da biomassa}

Os resultados da análise química imediata da biomassa estão na Tabela 1.

Tabela 1. Valores médios para os teores de carbono fixo, materiais voláteis e cinzas da biomassa de Bambu e de Eucalipto.

Table 1. Mean values of fixed carbon, volatiles and ash content of the Bamboo and Eucalyptus biomass.

\begin{tabular}{lccc}
\hline Espécie & Carbono Fixo (\%) & Teor de Voláteis (\%) & Teor de Cinzas (\%) \\
\hline B. tuldoides & $21,8 \mathrm{a}(0,6)$ & $75,2 \mathrm{a}(0,7)$ & $3,0 \mathrm{a}(0,1)$ \\
B. vulgaris var vittata & $22,8 \mathrm{ab}(0,3)$ & $74,7 \mathrm{a}(0,3)$ & $2,5 \mathrm{ab}(0,2)$ \\
D. asper & $23,0 \mathrm{~b}(0,4)$ & $75,0 \mathrm{a}(0,2)$ & $2,1 \mathrm{~b}(0,3)$ \\
E. urograndis & $17,5 \mathrm{c}(0,2)$ & $82,2 \mathrm{~b}(0,5)$ & $0,3 \mathrm{c}(0,1)$ \\
\hline
\end{tabular}

Médias seguidas de desvio padrão. Médias seguidas da mesma letra, na coluna, não diferem entre si pelo Teste de Tukey a 5\% de significância.

Os teores médios de carbono fixo, relacionados ao processo de liberação de energia para a combustão, foram de $21,8 \%$ para $B$. tuldoides, $22,8 \%$ para $B$. vulgaris var. vittata e $23,0 \%$ para $D$. asper, sendo estes superiores estatisticamente ao encontrado para o $E$. urograndis de $17,5 \%$. Combustíveis com alto índice de carbono fixo possuem queima mais lenta, implicando maior tempo de residência nos aparelhos de queima, em comparação com outros que tenham menor teor de carbono fixo 
(OLIVEIRA et al., 2010), o que indica, por meio dos resultados obtidos, uma vantagem das espécies de bambu estudadas em comparação à madeira de eucalipto.

Os teores de cinzas foram estatisticamente superiores na biomassa das espécies de bambu, com 3,0; 2,5 e 2,1\% para B. tuldoides, B. vulgaris var. vittata e D. asper, respectivamente, se comparados ao teor de cinza médio na biomassa do E. urograndis (0,3\%) e estão de acordo com os teores apresentados por Liu et al. (2014). Brito et al. (1987) encontraram um teor de cinzas sete vezes mais elevado para o Bambusa vulgaris em relação ao Eucalyptus urophylla.

De acordo com Liu et al. (2014) os elevados teores de cinzas das espécies de bambus se dão pelos altos teores de sílica presentes na composição química dos colmos. Em função da maior quantidade de cinzas ( 2 a 3\%) encontradas na biomassa das espécies de bambu em relação ao E. urograndis $(0,3 \%)$ faz-se necessário a utilização de caldeiras com grelhas rotativas, uma vez que o teor de cinzas é um parâmetro com relevância no design da caldeira e na operação da sua limpeza, pois a combustão de biomassa com elevados teores de cinza necessitará de um processo da sua remoção mais regular e eficaz, em função da sua abrasividade que, no longo prazo, pode causar corrosão nos elementos metálicos dos queimadores (CARNEIRO et al., 2013; LIU et al., 2014).

Os teores médios de materiais voláteis foram de $75,2 \%$ para B. tuldoides, $74,7 \%$ para B. vulgaris var. vittata, $75,0 \%$ para $D$. asper e $82,2 \%$ para o E. urograndis (estatisticamente superior), estando estes valores dentro da faixa esperada de 65 a 85\% (ARANTES et al., 2013). O teor de materiais voláteis está relacionado à queima no processo da carbonização, sendo esta mais rápida quanto maior o teor de voláteis. Scurlock et al. (2000), em estudo com espécies de bambu do gênero Phyllostachys, encontraram valores médios de $73,7 \%$ e $16,7 \%$ para $P$. nigra, 70,5\% e 15,7\% para $P$. bambusoides e $72,2 \%$ e $16,3 \%$ para $P$. bissetii, para teor de voláteis e carbono fixo, respectivamente.

Quanto ao PCS, os valores médios observados variaram entre 4515,1 a 4662,9 kcal.kg-1 e de acordo com a análise de variância, não se observa diferença significativa entre as espécies (Tabela 2). UEDA (1981) considerando a utilização de colmos de bambu para energia como muito importante, citou valores de poder calorífico de 4.500-5.400 kcal. $\mathrm{kg}^{-1}$. Sendo assim, a biomassa das espécies de bambu estudadas possui valores de PCS comumente mencionados na literatura especializada para a madeira de eucalipto para uso na queima direta (ARANTES et al., 2013; CARNEIRO et al., 2013).

Em relação à densidade básica da biomassa (Tabela 2), verifica-se que os valores médios de $B$. vulgaris var. vittata $\left(0,46 \mathrm{~g} . \mathrm{cm}^{-3}\right)$ foram similares aos encontrados para a madeira de eucalipto $(0,48$ g. $\left.\mathrm{cm}^{-3}\right)$, tendo sido observados valores médios estatisticamente inferior e superior para os colmos de B. tuldoides $\left(0,42 \mathrm{~g} . \mathrm{cm}^{-3}\right)$ e D. asper $\left(0,60 \mathrm{~g} \cdot \mathrm{cm}^{-3}\right)$, respectivamente. A alta densidade observada para o $D$. asper constitui uma vantagem para esta espécie, uma vez que para um mesmo volume de madeira pode-se obter maior rendimento gravimétrico em carvão vegetal se a densidade dos materiais utilizados for mais alta (OLIVEIRA et al., 2010).

Tabela 2. Valores médios de densidade básica $\left(\mathrm{g} . \mathrm{cm}^{-3}\right)$, poder calorífico $\left(\mathrm{kcal} . \mathrm{kg}^{-1}\right)$ e densidade energética $\left(\mathrm{Gcal} . \mathrm{m}^{-3}\right)$ da biomassa de Bambu e de Eucalipto.

Table 2. Mean values of specific gravity $\left(\mathrm{g} . \mathrm{cm}^{-3}\right)$, high heating value $\left(\mathrm{kcal} . \mathrm{kg}^{-1}\right)$ and energy density $\left(\mathrm{Gcal} . \mathrm{m}^{-3}\right)$ of the Bamboo and Eucalypt biomass.

\begin{tabular}{|c|c|c|c|}
\hline Espécie & $\begin{array}{c}\text { Densidade Básica } \\
\left(\text { g.cm }^{-3}\right)\end{array}$ & $\begin{array}{l}\text { Poder Calorífico } \\
\left.\text { (kcal.kg }{ }^{-1}\right)\end{array}$ & $\begin{array}{c}\text { Densidade energética } \\
\left(\text { Gcal. } \mathrm{m}^{-3}\right)\end{array}$ \\
\hline B. tuldoides & 0,421 a $(0,1)$ & 4515,1 a $(38,1)$ & 1,96 a $(0,1)$ \\
\hline B. vulgaris var vittata & 0,462 b $(0,1)$ & 4662,9 a $(45,2)$ & $2,16 \mathrm{~b}(0,1)$ \\
\hline D. asper & $0,604 \mathrm{c}(0,1)$ & 4526,2 a $(29,7)$ & $2,80 \mathrm{c}(0,2)$ \\
\hline E. urograndis & 0,482 b $(0,1)$ & 4657,6 a $(41,2)$ & $2,17 \mathrm{~b}(0,1)$ \\
\hline
\end{tabular}

Médias seguidas de desvio padrão. Médias seguidas da mesma letra, na coluna, não diferem entre si pelo Teste de Tukey a $5 \%$ de significância.

A densidade energética, que é o produto entre a densidade básica e o poder calorífico superior, foi estatisticamente superior nos colmos de $D$. asper em função da alta densidade básica observada para esta espécie de bambu, uma vez que o poder calorífico não diferiu entre as espécies estudadas.

\section{Caracterização energética do carvão vegetal}

Os resultados da análise química imediata e o valor médio do PCS do carvão vegetal estão na Tabela 3. 
Tabela 3. Valores médios para os teores de carbono fixo, materiais voláteis, cinzas e poder calorífico do carvão de Bambu e de Eucalipto.

Table 3. Mean values of fixed carbon, volatiles, ash content and higher heating value of the Bamboo and Eucalyptus charcoal.

\begin{tabular}{|c|c|c|c|c|}
\hline Espécie & $\begin{array}{c}\text { Carbono Fixo } \\
(\%)\end{array}$ & $\begin{array}{c}\text { Teor de Voláteis } \\
(\%)\end{array}$ & $\begin{array}{c}\text { Teor de Cinzas } \\
(\%)\end{array}$ & $\begin{array}{c}\text { Poder Calorífico } \\
\text { kcal.kg-1 }\end{array}$ \\
\hline B. tuldoides & $72,3 \mathrm{ab}(0,4)$ & 21,7 a $(0,3)$ & 6,1 a $(0,3)$ & 6752,1 a $(47,2)$ \\
\hline B. vulgaris var vittata & 73,4 b $(1,8)$ & 23,6 a $(1,7)$ & $3,0 \mathrm{~b}(0,2)$ & 6776,7 a $(62,6)$ \\
\hline D. asper & $71,5 \mathrm{ab}(0,5)$ & 26,5 b $(0,3)$ & $1,9 \mathrm{~b}(0,8)$ & 6640,5 a $(58,1)$ \\
\hline E. urograndis & 70,4 a $(0,2)$ & $29,1 \mathrm{C}(0,2)$ & $0,4 \mathrm{c}(0,3)$ & 6669,9 a $(41,9)$ \\
\hline
\end{tabular}

Os teores médios de carbono fixo do carvão das espécies de bambu foram de 72,3\% para B. tuldoides, 73,4\% para B. vulgaris var. vittata e de $71,5 \%$ para $D$. asper. Brito et al. (1987) encontraram valores médios de carbono fixo superiores para as espécies $B$. tuldoides e B. vulgaris var. vittata de, respectivamente, 90,4\% e 84,2\%, possivelmente em decorrência da maior temperatura de carbonização utilizada em seu estudo $\left(550^{\circ} \mathrm{C}\right)$. De acordo com Carneiro et al. (2013), os teores de carbono fixo no carvão são sensivelmente influenciados pela temperatura final do sistema de conversão, elevando-se rapidamente com o aumento da temperatura de carbonização, enquanto decrescem os teores de hidrogênio e oxigênio sendo, da mesma forma, acompanhado pela redução do teor de materiais voláteis.

Para o carvão de E. urograndis, o teor de carbono fixo médio foi de 70,5\%, valor similar aos encontrados para B. tuldoides e D. asper, e inferior ao encontrado para o B. vulgaris var. vittata, que apresentou o maior valor entre as espécies. Arantes et al. (2013) encontraram valor de carbono fixo de $72,8 \%$ para o mesmo clone e com a mesma temperatura final de carbonização. O carbono fixo é um dos mais importantes indicadores de qualidade do carvão vegetal como termorredutor na siderurgia, contribuindo para o aumento na produtividade dos altos-fornos, pois quanto maior o teor de carbono fixo do carvão maior será a utilização volumétrica do alto forno (CARNEIRO et al., 2013).

Em relação aos teores de voláteis, tem-se que os valores médios encontrados para as espécies de bambu foram significativamente inferiores ao encontrado para o eucalipto. De acordo com Santos (2008), embora maiores teores de voláteis impliquem em menores teores de carbono fixo, essa propriedade está relacionada à estabilidade da chama e à velocidade do processo de combustão. Dessa forma, os menores teores de voláteis para as espécies de bambu em relação ao eucalipto não representaram uma vantagem uma vez que não se relacionaram a maiores valores de carbono fixo, com exceção da espécie B. vulgaris var. vittata, mas se relacionam a elevada porcentagem de teor de cinzas para as três espécies de bambu, sendo estes valores significativamente superior ao encontrado para o carvão de eucalipto, com o maior valor para a espécie B. tuldoides $(6,09 \%)$.

A presença de cinzas compromete a qualidade do carvão vegetal, principalmente quanto ao seu uso na siderurgia, uma vez que causa desgaste no alto forno e pode comprometer a qualidade do ferro-gusa, com consequente formação de trincas e fissuras (NEVES et al., 2011; CARNEIRO et al., 2013).

Quanto ao PCS, os valores médios observados para o carvão de B. tuldoides, B. vulgaris var. vittata, D. asper e E. urograndis foram de 6752,1;6776,7;6640,5 e 6669,9 kcal.kg-1, respectivamente, sem diferença estatística. Santos (2008) e Soares et al. (2015) relataram que o poder calorífico superior

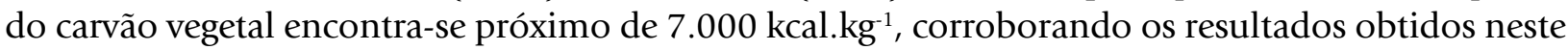
trabalho, sendo o principal parâmetro para expressar a capacidade de geração de energia em substituição aos combustíveis derivados do petróleo. O incremento dos valores de poder calorífico no carvão vegetal em relação à biomassa $\left(4515,1\right.$ a $\left.4662,9 \mathrm{kcal}^{\mathrm{kg}}{ }^{-1}\right)$ foi na ordem de $44 \%$.

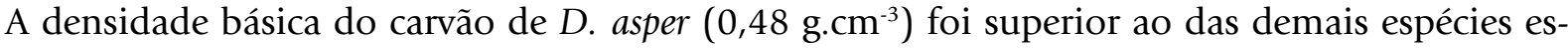
tudadas $\left(0,32\right.$ a $\left.0,36 \mathrm{~g}_{\mathrm{cm}} \mathrm{cm}^{-3}\right)$, em função da maior densidade observada nos colmos desta espécie (Tabela 4). Segundo Botrel et al. (2007), materiais de densidades mais elevadas produzem carvão com maior densidade, o que pode ser constatado neste trabalho.

Os valores de densidade energética encontrados para o carvão vegetal de B. tuldoides; $B$. vulgaris var. vittata, D. asper e E. urograndis foram de 2,16; 2,30; 3,19 e 2,40 Gcal.m ${ }^{-3}$, respectivamente, sendo estatisticamente superior para a espécie $D$. asper em função da elevada densidade observada no carvão. A densidade energética é um importante parâmetro para combustíveis sólidos, pois, avalia 
a quantidade de energia armazenada em um determinado volume de material. No caso do carvão vegetal, os valores de densidade energética são superiores aos da biomassa (Tabela 2) em função do aumento do poder calorífico no material pirolisado, apesar da redução da densidade nos carvões.

Tabela 4. Densidade básica, densidade energética e rendimento do carvão de Bambu e de Eucalipto.

Table 4. Specific gravity, energy density and yield of the Bamboo and Eucalypt charcoal.

\begin{tabular}{lccc}
\hline Espécie & $\begin{array}{c}\text { Densidade Básica } \\
\left(\mathbf{g} \cdot \mathbf{c m}^{-3}\right)\end{array}$ & $\begin{array}{c}\text { Densidade energética } \\
\left(\mathbf{G c a l} . \mathbf{m}^{-3}\right)\end{array}$ & $\begin{array}{c}\text { Rendimento em Carvão } \\
\mathbf{( \% )}\end{array}$ \\
\hline B. tuldoides & $0,32 \mathrm{~b}(0,1)$ & 2,16 a $(0,2)$ & 34,1 a $(1,0)$ \\
B. vulgaris var vittata & $0,34 \mathrm{~b}(0,1)$ & 2,30 a $(0,2)$ & 34,7 a $(0,7)$ \\
D. asper & 0,48 a $(0,2)$ & $3,19 \mathrm{~b}(0,3)$ & $36,9 \mathrm{~b}(1,1)$ \\
E. urograndis & $0,36 \mathrm{~b}(0,1)$ & 2,40 a $(0,2)$ & $30,8 \mathrm{c}(1,0)$ \\
\hline
\end{tabular}

Médias seguidas de desvio padrão. Médias seguidas da mesma letra, na coluna, não diferem entre si pelo Teste de Tukey a $5 \%$ de significância.

De acordo com os resultados obtidos e observados na Tabela 4, o valor médio do rendimento gravimétrico foi de 36,9\% para $D$. asper, 34,7\% para B. vulgaris var. vittata, 34,1\% para $B$. tuldoides e $30,8 \%$ para E. urograndis. O rendimento em carvão para B. vulgaris var. vittata foi superior ao encontrado por Brito et al. (1987), que obtiveram um valor de $29,1 \%$ de rendimento gravimétrico para a mesma espécie, porém em uma temperatura máxima média de $550^{\circ} \mathrm{C}$ e o do E. urograndis similar ao observado por Assis et al. (2012) nas mesmas condições de carbonização (31,6\%).

Nas mesmas condições de carbonização o rendimento em carvão das espécies de bambu foi superior ao da madeira de eucalipto. Além da taxa de aquecimento e da temperatura final de carbonização, o rendimento gravimétrico e as demais características energéticas são influenciadas pela composição química da biomassa. Neste sentido, recomenda-se a realização da determinação dos teores de lignina e de holocelulose para uma melhor explicação do comportamento das espécies de bambu em relação ao eucalipto.

Cabe ressaltar a necessidade da realização de pesquisas complementares relacionados as espécies de bambu, principalmente no que se refere a produtividade, disponibilidade e acesso à biomassa, visando a indicação do seu uso como fonte energética alternativa.

\section{CONCLUSÕES}

- A biomassa e o carvão vegetal das espécies de bambu apresentaram características energéticas similares ou superiores em relação ao híbrido de Eucalyptus grandis $x$ Eucalyptus urophylla, exceto para os teores de materiais voláteis e de cinzas.

- As espécies Bambusa vulgaris var. vittata, Dendrocalamus asper e Bambusa tuldoides apresentaram características energéticas que indicam o seu potencial para o uso como fonte de energia.

\section{AGRADECIMENTOS}

Ao CNPq pelo financiamento desta pesquisa - chamada nº 66/2013 - MCTI/AÇÃO TRANSVERSAL, processo $\mathrm{n}^{\circ} 458300 / 2013-6$.

\section{REFERENCIAS BIBLIOGRÁFICAS}

ABNT - ASSOCIAÇÃO BRASILEIRA DE NORMAS TÉCNICAS. NBR 8112/83: Carvão vegetal - Análise imediata. São Paulo, 1983.

ABNT - ASSOCIAÇÃO BRASILEIRA DE NORMAS TÉCNICAS. NBR 8633/84: Carvão vegetal - Determinação do poder calorífico. São Paulo, 1984.

ABNT - ASSOCIAÇÃO BRASILEIRA DE NORMAS TÉCNICAS. NBR 11941/2003: Determinação da densidade básica. São Paulo, 2003. 
ARANTES, M. D. C; TRUGILHO, P. F; SILVA, J. R. M.; ANDRADE, C. R. Características do carvão de um clone de Eucalyptus grandis W. Hill ex Maiden x Eucalyptus urophylla S. T. Blake. Cerne, Lavras, v. 19, n. 3, p. 423-431, jul./set. 2013.

ASSIS, M. R.; PROTÁSIO, T. P.; ASSIS, C. O.; TRUGILHO, P. F.; SANTANA, W. M. S. Qualidade e rendimento do carvão vegetal de um clone híbrido de Eucalyptus grandis x Eucalyptus urophylla. Pesquisa Florestal Brasileira, Colombo, v. 32, n. 71, p. 291-302, jul./set. 2012.

BARTLETT, M. S. Properties of sufficiency and statistical tests. Proceedings of the Royal Society, Series A, v. 160, p. 268-282, 1937.

BOTREL, M. C. G.; TRUGILHO P. F.; ROSADO, S. C. S.; SILVA, J. R. M. Melhoramento genético das propriedades do carvão vegetal de Eucalyptus. Revista Árvore, Viçosa, v. 31, n. 3, p. 391-398, 2007.

BRASIL. Lei $\mathrm{n}^{\circ} 12.484$ de 2011 - Política Nacional de Incentivo ao Manejo Sustentado e ao Cultivo do Bambu, 2011. Disponível em: < http://www.planalto.gov.br/ccivil_03/_ato2011-2014/2011/lei/l12484.htm>. Acesso em: 10 jun 2015.

BRITO, J. O.; TOMAZELLO FILHO, M.; SALGADO, A. L B. Produção e caracterização do carvão vegetal de espécies e variedades de bambu. IPEF, Piracicaba, n. 36, p. 13-17, 1987.

CARNEIRO, A. C. O. Pirólise lenta da madeira para produção de carvão vegetal. In: SANTOS, F.; COLODETTE, J.; QUEIROZ, J. H. (Ed.) Bioenergia e biorrefinaria - Cana-de-açúcar e Espécies Florestais. Viçosa: UFV, 2013. p. $429-455$

COSTA, T. M. S. Estudo da viabilidade técnica do emprego do bambu da espécie Bambusa vulgaris Schrad. como carvão vegetal. 2004. 74 p. Dissertação (Mestre em Ciências na área de Tecnologia Nuclear-Materiais) - Instituto de Pesquisas Energéticas e Nucleares, Autarquia Associada à Universidade de São Paulo, São Paulo.

EPE - EMPRESA DE PESQUISA ENERGÉTICA. Balanço Energético Nacional 2015: ano base 2014. Rio de Janeiro. 2015. 292 p.

HIDALGO-LÓPEZ, O. Bamboo: The gift of the gods. Colombia: D’Vinni Ltda, 2003. p. 142-153

IPCC - INTERGOVERNMENTAL PANEL ON CLIMATE CHANGE. Managing the risks of extreme events and disasters to advance climate change adaption. New York: Cambridge University Press, 2012. 582 p.

LEVENE, H. Robust Tests for the equality of variance. In: OLKIN, I. (Ed.) Contributions to Probability and Statistics, Palo Alto: Stanford University Press, 1960. p. 278-292.

LIU, Z.; FEI, B.; JIANG, Z.; CAI, Z.; LIU,X. Important properties of bamboo pellets to be used as commercial solid fuel in China. Wood Science and Technology, v. 48, n. 5, p. 903-917. 2014.

MOREIRA, A. C. O. Caracterização de Bambusa vulgaris Schrad. EX J. C. WENDL. var. vulgaris e dos resíduos de caldeira no processo de conversão térmica de energia. 2012. 61 p. Dissertação (Mestrado em Ciências Florestais). Universidade de Brasília. 2012.

NEVES, T. A.; PROTÁSIO, T. P.; COUTO, A. M.; TRUGILHO, P. F.; SilVA, V. O.; VIEIRA, C. M. M. Avaliação de clones de Eucalyptus em diferentes locais visando à produção de carvão vegetal. Pesquisa Florestal Brasileira, Colombo, v. 31, n. 68, p. 319-330. 2011

NOBRE, C. A.; SALAZAR, L. F.; OYAMA, M.; CARDOSO, M.; SAMPAIO G.; LAPOLA, D. Mudanças Climáticas e possíveis alterações nos Biomas da América do Sul. São Paulo: MMA. 2007. 29 p. Relatório n ${ }^{\circ} 06$. 
Santos et al. - Potencial de espécies de Bambu como fonte energética

OliveirA, A. C.; CARNEIRO, A. C. O.; VITAL, B. R.; ALMEIDA, W.; PEREIRA, B. L. C.; CARDOSO, M. T. Parâmetros de qualidade da madeira e do carvão vegetal de Eucalyptus pellita F. Muell. Scientia Forestalis, Piracicaba, v. 38, n. 87, p. 431-439, set. 2010.

SCHAEFFER, R., SZKLO, A. S.; LUCENA, A. F. P.; SOUZA, R. R.; BORBA, B. S. M. C.; COSTA, I. V. L.; PEREIRA JR., A. O.; CUNHA, S. H. F. Mudanças climáticas e segurança energética no Brasil. Rio de Janeiro: UFRJ. 2008. 35 p. Relatório Coppe.

SCURLOCK, J. M. O.; DAYTON, D. C.; HAMES, B. Bamboo: an overlooked biomass resource? National Renewable Energy Laboratory. 2000. 34 p.

SHAPIRO, S. S.; WILK, M. B. An analysis of variance test for normality (complete samples), Biometrika, Cambridge, v. 52, n. 3-4, p. 591-611, 1965.

SOARES, V. C.; BIANCHI, M. L.; TRUGILHO, P. F.; HÖFLER, J.; PEREIRA, A. J. Análise das propriedades da madeira e do carvão vegetal de híbridos de eucalipto em três idades. Cerne, Lavras, v. 21, n. 2, p. 191-197, 2015.

TRUONG, A. H.; LE, T. M. A. Overview of bamboo biomass for energy production. Hanoi: USTH, 2014.25 p.

TUKEY, J. W. Exploratory data analysis. Reading: Addison-Wesely, 1977.

UEDA, K. Bamboo industry in Japan, present and future. In: IUFRO WORLD CONGRESS, 17., Kyoto, 1981. Proceedings... Kyoto: Wood Research Institute/Kyoto University, 1981. p. 2-14.

VARANDA, L. D.; CARASCHI, J. C.; ALMEIDA FILHO, F. Potencial energético do carvão vegetal de bambu. In: ENCONTRO BRASILEIRO EM MADEIRAS E EM ESTRUTURAS DE MADEIRA, 12., 2010, Lavras. Anais... Lavras: UFLA, 2010.

Recebido em 20/10/2015

Aceito para publicação em 24/02/2016 